\title{
Structural characterization of different TRPA1 inhibitors
}

\author{
L Rouge $^{1}$ \\ ${ }^{1}$ Genentech \\ lionelr@gene.com
}

Mammalian transient receptor potential (TRP) channels control cell homeostasis by mediating Ca2+ across membranes in response to physical and chemical environmental stimuli. TRPA1 has been a target of interest for the pharmaceutical industry for its implication in pain and neurogenic inflammation. It is mostly expressed by primary afferent nociceptors and detects a variety of compounds through covalent modification of conserved cysteine and lysine residues. Because of its therapeutic value, an internal program was launched to identify specific TRPA1 inhibitors: this campaign led to the discovery of multiple interesting chemotypes. In order to guide optimization of these molecules, an effort to structurally enable TRPA1 for structure based drug design was initiated based on a previously published cryo-EM structure (Paulsen et al., Nature, 2015). A combination of protein engineering, biochemistry and cryo-EM approaches led to the establishment of a robust system (e.g. improved resolution, high reproducibility) which allowed us to identify a novel binding site and optimize molecules with improved properties.

Acta Cryst. (2020). A76, a126 